
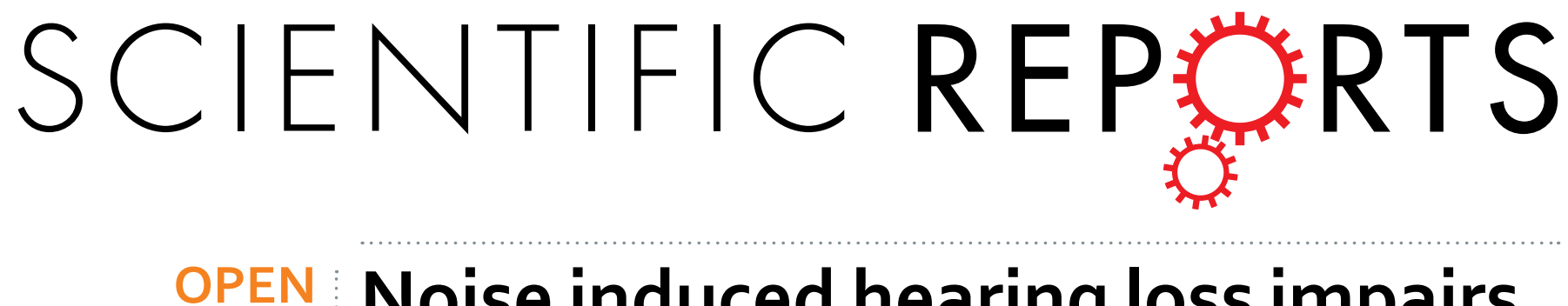

\title{
Noise induced hearing loss impairs spatial learning/memory and hippocampal neurogenesis in mice
}

Received: 14 September 2015

Accepted: 23 December 2015

Published: 04 February 2016
Lijie Liu ${ }^{1}$, Pei Shen ${ }^{1}$, Tingting He ${ }^{1}$, Ying Chang ${ }^{1}$, Lijuan Shi ${ }^{1}$, Shan Tao ${ }^{1}$, Xiaowei $\mathrm{Li}^{1}$, Qingying Xun ${ }^{1}$, Xiaojing Guo ${ }^{1,2}$, Zhiping $\mathrm{Yu}^{3}$ \& Jian Wang ${ }^{1,3}$

Hearing loss has been associated with cognitive decline in the elderly and is considered to be an independent risk factor for dementia. One of the most common causes for acquired sensorineural hearing loss is exposure to excessive noise, which has been found to impair learning ability and cognitive performance in human subjects and animal models. Noise exposure has also been found to depress neurogenesis in the hippocampus. However, the effect is mainly attributed to the oxidant stress of noise on the cognitive brain. In the present study, young adult CBA/CAJ mice (between 1.5 and 2 months of age) were briefly exposed a high sound level to produce moderate-to-severe hearing loss. In both the blood and hippocampus, only transient oxidative stress was observed after noise exposure. However, a deficit in spatial learning/memory was revealed 3 months after noise exposure. Moreover, the deficit was correlated with the degree of hearing loss and was associated with a decrease in neurogenesis in the hippocampus. We believe that the observed effects were likely due to hearing loss rather than the initial oxidant stress, which only lasted for a short period of time.

Hearing loss is one of the most common neurological disorders, affecting $10 \%$ of the general population ${ }^{1}$ and more than $40 \%$ of those over 60 years of age $\mathrm{e}^{2,3}$. Noise exposure is one of the major causes of acquired sensorineural hearing loss in adults ${ }^{4}$. However, the damaging effects of noise are not limited to the auditory system but rather extend to many other systems ${ }^{5}$. Recent studies have discussed the noise-related impairment of learning ability and cognitive performance ${ }^{6-9}$. Soldiers who were exposed to excessive noise levels, including explosions and blast waves, experienced severe noise-induced hearing loss (NIHL) and tinnitus ${ }^{10,11}$ as well as cognitive deficits and memory impairment ${ }^{12}$.

The mechanisms underlying the decline of cognitive functions after noise exposure are not entirely clear. Animal studies have suggested that noise exposure is likely to impair cognitive functions through two different but closely related approaches. One is due to the oxidative reaction initiated by noise exposure. Many studies have shown that increased oxidative stress is the cause of neuronal degeneration in many auditory nuclei as well as the brain regions critical for cognitive functions ${ }^{6,7,13,14}$. Another potential effect of noise exposure is a change in the auditory input to the cognitive brain after NIHL. Overall, this effect has largely been ignored in studies of the impact of noise on cognitive function and neurogenesis; however, its existence is supported by the connection between the auditory brain and cognitive brain ${ }^{15}$, the hippocampal degeneration and deterioration of spatial memory observed in CBA/CAJ mice with age-related hearing $l o s s^{16}$, and the suppression of hippocampal neurogenesis observed in the rats long after unilateral NIHL ${ }^{17}$.

In the present study, CBA/CAJ mice were exposed to noise at a high level (123 dB SPL) but for a brief period of time $(2 \mathrm{~h})$ to produce permanent hearing loss. Cognitive performance and hippocampal neurogenesis were examined 3 months after noise exposure, long after the disappearance of oxidative stress. The long delay between the presence of oxidative stress and cognitive examination allowed for the investigation of the role of NIHL, rather than oxidative stress, in the decline of hippocampal learning/memory function and neurogenesis.

${ }^{1}$ Department of Physiology, Medical College of Southeast University, Nanjing China. ${ }^{2}$ Children's medical center, the Second Affiliated Hospital of Nanjing Medical University, Nanjing, China. ${ }^{3}$ School of Human Communication Disorders, Dalhousie University, Halifax, Canada. Correspondence and requests for materials should be addressed to L.L. (email: liulj321@163.com) or J.W. (email: Jian.Wang@dal.ca) 


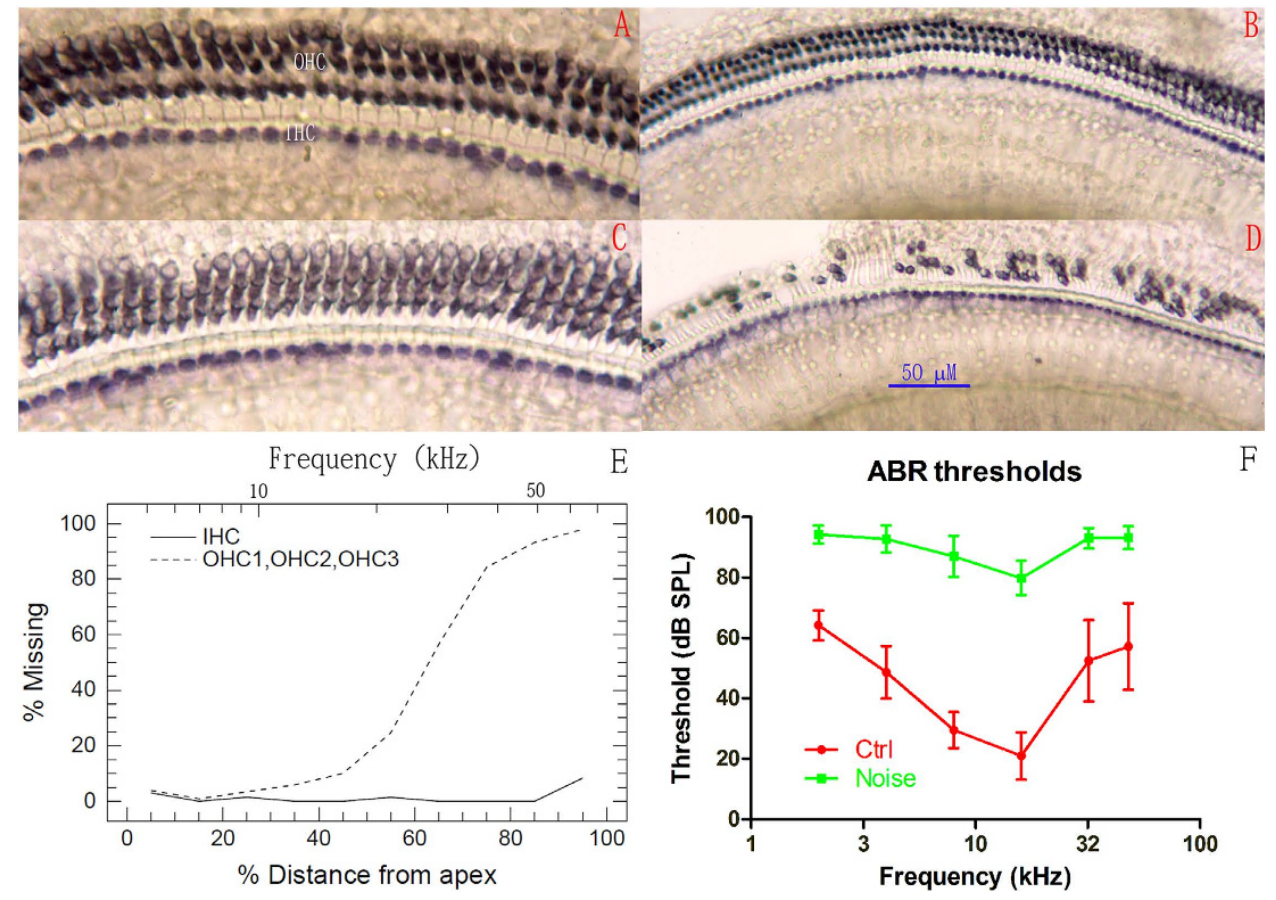

Figure 1. Noise-induced hair cell loss and ABR threshold shift measured 3 months after the noise exposure at 6-8 weeks of age. (A-D) Representative images of SDH staining of HCs from the two groups. (A,B) Apical and basal turns, respectively, in the control group; (C,D) Apical and basal turns, respectively, in the noise group. Massive OHC loss is clearly observed in (D). (E) Cochleogram of the noise-exposed animals showing the loss of OHCs concentrated in the basal half of the cochlea. (F) ABR thresholds. The differences between the two groups show that moderate-to-severe hearing loss was established in the noise group.

\section{Results}

Noise-induced hearing loss. Figure 1A-D shows representative images of succinate dehydrogenase (SDH) staining on both outer and inner hair cells (OHCs and IHCs) from the two groups. Two images were presented for each group, one from the apical turn (Fig. 1A,C) and the other from the basal turn (Fig. 1B,D). In contrast to the spread of hearing loss on the ABR audiogram, it is clear that $\mathrm{HC}$ loss was limited to the basal half of the cochlea, mainly in OHCs. This is further demonstrated in the cochleogram of HC loss in Fig. 1E.

ABR audiograms were obtained at the age of 4.5-5 months and compared between the control group and the noise-exposed group to determine differences in hearing sensitivity. Figure $1 \mathrm{~F}$ shows that the ABR thresholds of the noise group were much higher than those of the control group at every frequency tested. The frequency-averaged threshold was found to be $89.972 \pm 2.907 \mathrm{~dB}$ SPL in the noise group, which was significantly higher than the value of $45.5 \pm 6.995 \mathrm{~dB}$ SPL found for the control group (mean $\pm \mathrm{SD}, \mathrm{n}=30$; Student $\mathrm{t}$ test, $\mathrm{t}=32.156, \mathrm{p}<0.001$ ). The mean difference of $44.47 \mathrm{~dB}$ between the two groups suggests a moderate-to-severe degree of hearing loss in the noise group.

Impact of NIHL on spatial learning and memory. Spatial learning ability and memory was tested in a Morris water maze (MWM). The first stage of this test involved a five-day training period in which the mice learned to find the hidden platform underwater (measured as escape latency) using spatial cues around the pool. Figure 2A compares the changes in escape latency during the training days between the two groups. The figure demonstrates slower learning in the noise group. A two-way ANOVA with the factors of noise exposure and days of training revealed a significant effect of noise exposure $(\mathrm{F} 1=7.229, \mathrm{p}<0.008)$ on escape latency. Post-hoc pairwise comparisons (Tukey method) showed that the escape latency of the noise group was significantly longer than that of the control group on days 1,2 and 3 of training (indicated by the asterisks in Fig. $2 \mathrm{~A}$ ). The second stage of the MWM was the spatial orientation test, in which the hidden platform was removed, and the number of crosses through the platform area within 60 seconds was counted as an index of the spatial memory of the platform. This number was $3.00 \pm 0.32$ in the noise group, which is significantly lower than the value of $4.23 \pm 0.35$ obtained in the control group (one tail Student $t$ test, $t=2.946, \mathrm{n}=22$ in each group, $p<0.05$, Fig. 2B).

Correlation between NIHL and MWM results. A Pearson correlation analysis was performed to verify whether the degree of NIHL is correlated to the outcome of MWM tests. A significant positive correlation was observed between the frequency-averaged $\mathrm{ABR}$ threshold and the averaged escape latency over the 5 days of training for each individual (Fig. $2 \mathrm{C}, \mathrm{r}=0.524, \mathrm{p}=0.001$ ). In addition, there was a negative correlation between the frequency-averaged ABR threshold and the spatial memory manifested by the platform crossing times in the spatial orientation experiment (Fig. $2 \mathrm{D}, \mathrm{r}=-0.4, \mathrm{p}=0.004$ ). 

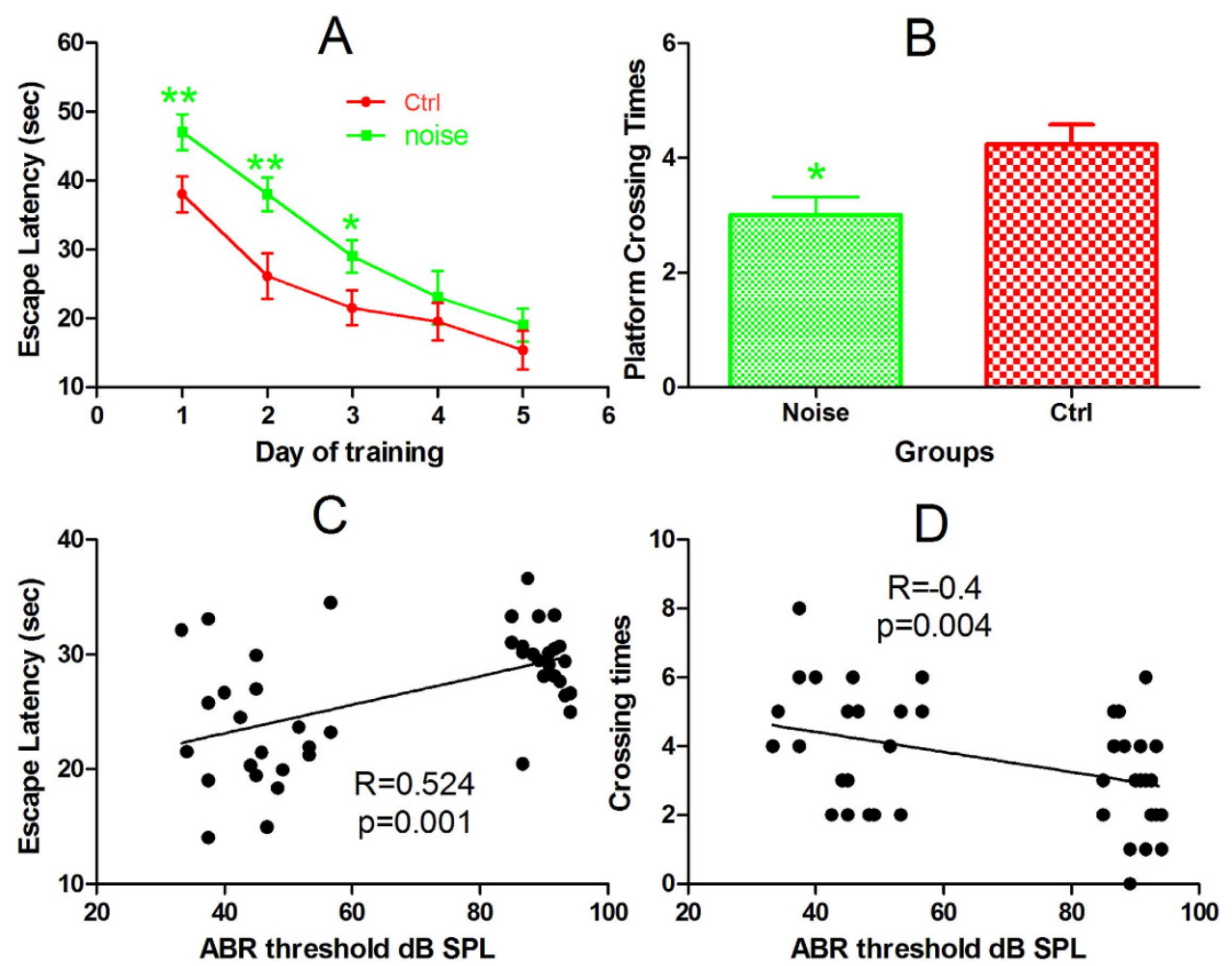

Figure 2. Effect of NIHL on MWM performance measured 3 months after the noise exposure at 6-8 weeks of age. (A) Change in escape latency with training days, (B) Platform crossing time in 60 second probe test. ${ }^{* *} \mathrm{p}<0.01,{ }^{*} \mathrm{p}<0.05$. (C) Correlation between ABR threshold and escape latency, (D) Correlation between ABR threshold and platform crossing time. Inserted lines are the results of linear regressions.

Oxidative Stress. Figure 3A shows the change in serum corticosterone (CORT) level after noise exposure. A one-way ANOVA was performed and showed that the serum CORT level $(224.44 \pm 10.63 \mathrm{ng} / \mathrm{ml})$ was significantly higher in the noise group than in the control group, as also shown by the post-hoc pairwise tests; however, this difference was only observed immediately after noise exposure, suggesting a transient effect of noise on CORT level. The changes in superoxide dismutase (SOD, Fig. 3B) and malondialdehyde (MDA, Fig. 3C) levels in the hippocampus were also transient (recovered within 1 month after noise exposure), and no significant change in ROS/RNS (Fig. 3D, measured by DCF fluorescence) was observed.

Neurogenesis in the hippocampus. The impact of NIHL on neurogenesis was evaluated by counting newly generated hippocampal cells in the dentate gyrus (DG) region using antibodies against DCX (a marker specific to neurons newly generated within the last 2-3 weeks) and Ki67 (a marker of proliferation). The representative images for both DCX (Fig. 4A,B) and Ki67 staining (Fig. 5A,B) indicate a decrease in neurogenesis in the noise group. Figures 4C and 5C compare the average counts of DCX/Ki67 positive cells in the whole DG region in both groups. Two-way ANOVAs were performed for both DCX and Ki67 cell counts against the factors of noise and training in the MWM. A significant effect of noise was observed for both the DCX and Ki67 cell counts $(\mathrm{F} 1=22.768, \mathrm{p}<0.001$ for DCX cells and F1 $=23.228, \mathrm{p}<0.001$ for Ki67 cell counts) but not for the factor of training (no difference between the trained and non-trained subjects in either the noise or control groups). The pooled number of DCX positive cells of the control group was $5756 \pm 208$, which was significantly higher than that of the noise group: $4387 \pm 191(\mathrm{t}=4.844, \mathrm{p}<0.0001)$. Similarly, the pooled numbers of Ki67 cells were $854 \pm 30$ and $668 \pm 22$ for the control and the noise groups, respectively, and the difference was statistically significant $(\mathrm{t}=5.115, \mathrm{p}<0.0001)$.

\section{Discussion}

In the present study, young adult CBA/CAJ mice were briefly exposed to noise, producing NIHL, which was verified to be of a moderate-severe degree by comparing the ABR threshold between the two groups at three months after noise exposure (Fig. 1E). Because the highest sound level in the ABR test is $90 \mathrm{~dB}$ SPL, the threshold over this level was assigned as $95 \mathrm{~dB}$ SPL. This ceiling effect was likely to cause an under-estimation of the threshold in the noise group. The effect of oxidative stress produced by the noise exposure was transient, as indicated by the changes in serum CORT level and the levels of three commonly used oxidative detection agents in the hippocampus (Fig. 3). The MWM test at this age showed that the animals in the noise group were slower in spatial learning and poorer in spatial memory (Fig. 2). Performance in the MWM appeared to be well correlated with hearing threshold (Fig. 2C,D). Furthermore, the NIHL appeared to decrease the level of neurogenesis in the hippocampus. 

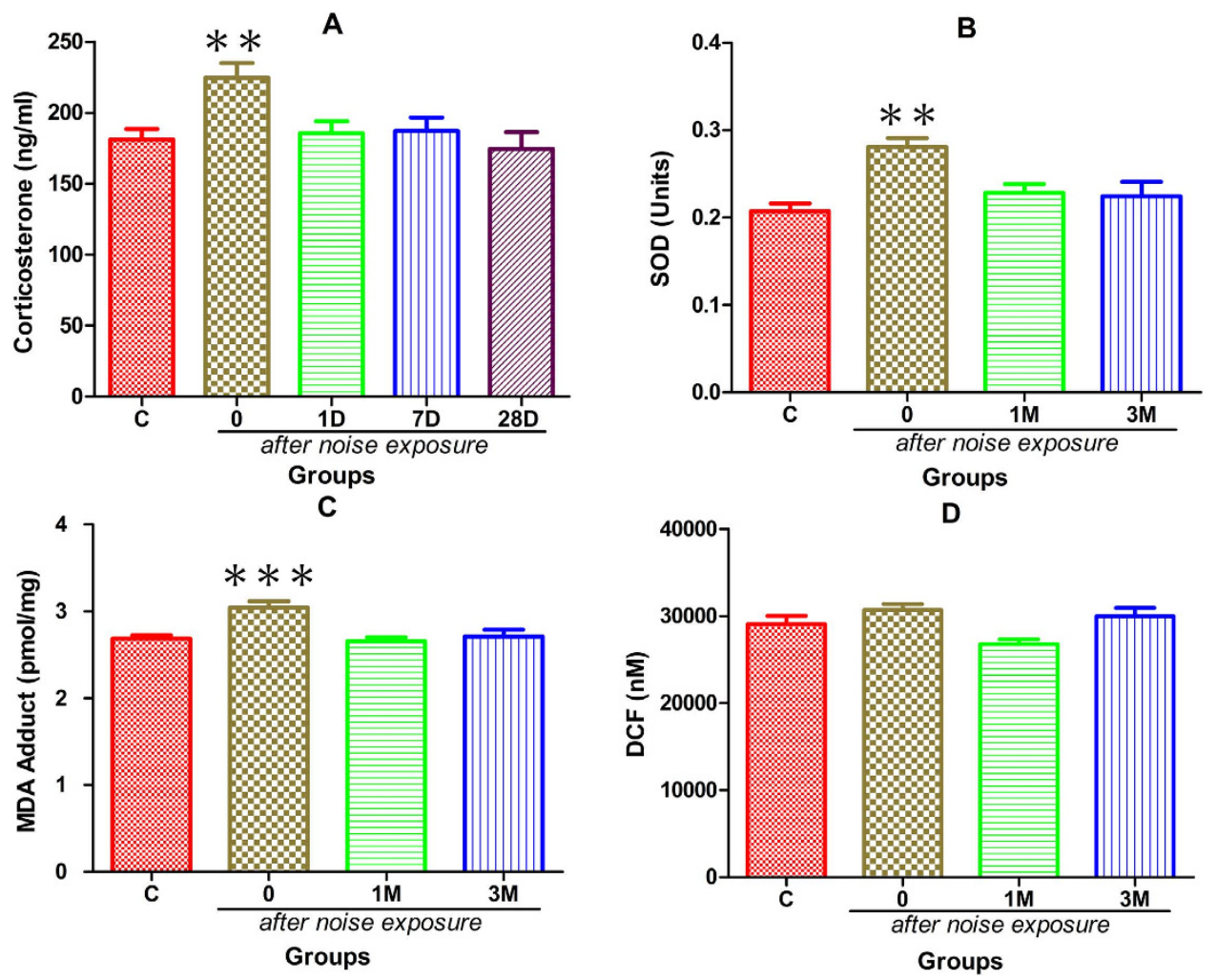

Figure 3. Effects of noise exposure on oxidative stress measured at different time points after noise exposure at 6-8 weeks of age. The time points of the evaluation are indicated in the figure (d: days, M: month after the noise exposure) (A) Plasma CORT hormone levels, (B) SOD activity, (C) MDA adduct content, (D) DCF level in hippocampus. The quantity of ROS/RNS was calculated based on the measure of DCF fluorescence (see methods section for details). (Mean \pm S.E.M., $n=8,{ }^{* *} \mathrm{p}<0.01,{ }^{* *} \mathrm{p}<0.001$ ).

The adverse impact of noise on learning and memory and hippocampal neurogenesis has been reported by many previous studies ${ }^{5,9,18-23}$. Most of those previous studies focused on the oxidative stress induced by noise; thus, in those studies, the cognitive functions and changes in brain morphology and molecular content were observed shortly after or even during the period of noise exposure, when the oxidative stress response was strong. The possibility of NIHL, rather than noise-induced stress, having an effect on cognitive function has largely been ignored. In fact, in many previous studies, the noise level was so low that hearing loss, in terms of changes in hearing sensitivity, was not expected to occur and was therefore not even documented. For example, impaired learning and memory capabilities were found in mice after exposure to white noise at $80 \mathrm{~dB}$ SPL 2 hours per day for a 6-week period, and the deterioration in learning and memory caused by this noise exposure was attributed to the increased level of MDA and SOD detected in the inferior colliculus, auditory cortex and hippocampus ${ }^{6}$. Similar results were reported in other studies that investigated noise exposure at higher levels (100 dBA for 4 hours daily for 30 days $)^{24}$.

While the role of noise-induced stress in cognitive impairment and neurogenesis is widely recognized, the independent effect of NIHL should also be considered, especially in cases of brief noise exposure. We think that the stress induced by the brief noise exposure in the present study is unlikely the reason for the deteriorated cognitive function and the depressed neurogenesis measured three months after the noise exposure. This argument is based upon several reasons. First of all, it has been recognized that adrenal glucocorticoid hormones are common pathway to the negative impact of stress on neurogenesis and related cognitive functions ${ }^{25-27}$. This connection has been demonstrated by (1) that cells in hippocampus express high level of glucocorticoid (GC) receptors ${ }^{28,29}$, which make them sensitive to GC mediated damage, (2) that administration of exogenous GCs decreases neurogenesis, and (3) that blockage of GCs prevents stress induced depression on neurogenesis ${ }^{30}$. Secondly, the impact of acute (brief) stresses of many different types on stress hormones is transient: it occurs in hours or days and quickly recovered after exposure to stressor ${ }^{30-32}$. The GC level is under restrictive regulation to which hippocampus neurons actively participate: the newly generated neurons here play a critical role in appropriate shut-off of the stress induced GC responses ${ }^{33,34}$. This is also true in the oxidative stress induced by brief noise exposure reported by others ${ }^{35,36}$ and in the present experiment. Thirdly, in most cases, long-lasting detectable changes in neurogenesis occur after prolonged and severe stress exposure ${ }^{32}$. Lastly, limited but significant data are available showing that the effect of transient noise exposure is transient. For example, spatial memory (similar to what we examined) was found to be deteriorated in a temporal pattern after impulse noise exposure at $165 \mathrm{~dB}$ SPL in rats: a totally recovery was seen within 24 hours after the noise ${ }^{18}$. In another study, a brief exposure to tone of $16 \mathrm{kHz}$ at $110 \mathrm{~dB}$ SPL for 1 hour failed to show any changes in various behavioral tests for learning and memory ${ }^{37}$. 

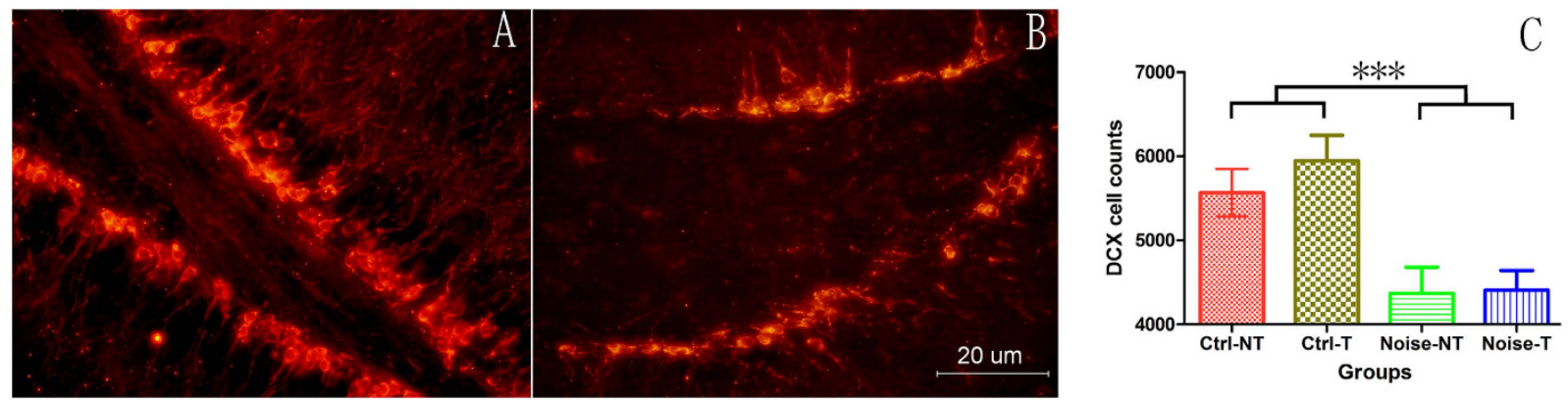

Figure 4. Comparison of DCX cells. (A) Representative images of the control group and (B) noise group. (C) Statistical comparison. ${ }^{* *} \mathrm{p}<0.001$ (between the noise and control groups).
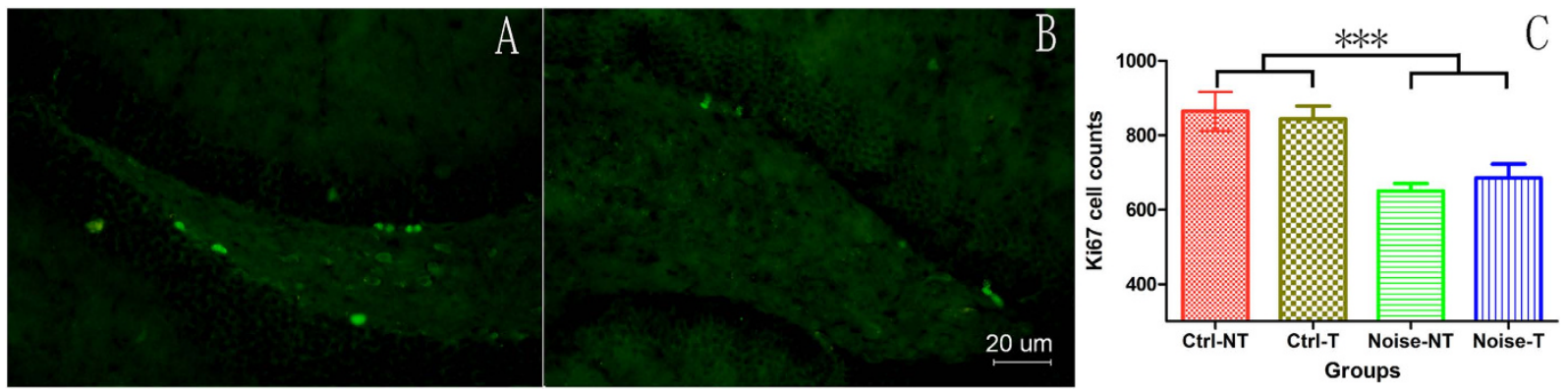

Figure 5. Comparison on Ki67 cells 3 months after noise exposure at 6-8 weeks of age. (A) Representative images of the control group and (B) noise group. (C) Statistical comparison. ${ }^{* * *} \mathrm{p}<0.001$ (between the noise and control groups).

To further confirm the contribution of negative impact of hearing loss, the cognitive functions and neurogenesis should be evaluated by using conditional knockout mouse models in which hearing loss can be produced in adulthood with no or minimal stress. However, this does not devalue the noise model because NIHL impacts a larger population.

The possible impact of NIHL (rather than oxidative stress) on cognitive function is also supported by several lines of previous research data. Firstly, recent studies have demonstrated that hearing loss in general is an independent risk factor of dementia ${ }^{38-41}$; however, exactly how hearing loss promotes the development of dementia still has to be investigated. Secondly, a strong anatomical and functional connection exists between brain regions in terms of auditory and cognitive functions. The hippocampus receives auditory input through the lemniscal ascending pathway, which transmits acoustic stimuli from the inferior colliculus to the auditory cortex and then to the hippocampus ${ }^{42}$. Furthermore, the hippocampus projects indirectly to the auditory cortex ${ }^{43}$. The auditory association cortex has both direct and indirect pathways to the hippocampus and receives its' indirect input. These connections enable the formation of long-term auditory memories and facilitate the processing of linguistic and musical input ${ }^{15}$. Thirdly, reduced auditory input after peripheral hearing loss has been reported to cause hippocampal degeneration and impaired memory function. For example, CBA/CAJ mice with age-related HL demonstrated hippocampal degeneration and had reduced spatial memory, as indicated by the results of the MWM test ${ }^{16}$. Even in cases where no hearing loss occurred, as indicated by the lack of change in hearing sensitivity (such as that reported in some previous studies that addressed the effect of noise on cognitive function), reduced auditory input from the peripheral hearing organ to the brain is expected based on recent studies of 'hidden' noise-induced hearing loss. Noise exposure producing no permanent threshold shift can largely damage the synapses between inner hair cells and the primary auditory neuron and therefore reduce the output of the cochlea to the brain ${ }^{44-46}$. Finally, the possible impact of $\mathrm{HL}$ on cognitive function is supported by the correlation of auditory threshold and the score of MWM performance.

A significant reduction in cell proliferation and neuronal generation was observed in the noise group, similar to that reported by other researchers ${ }^{17,36,37,47}$. Our study took the additional step of linking the decrease in hippocampal neurogenesis with the poorer performance in regard to spatial learning and memory during training in adult animals. However, we did not find any impact of training on the number of DCX or Ki67 cells in either the control or noise group. This result is inconsistent with the fact that the major effect of training is on the rescue of cells generated within one to two weeks prior ${ }^{48-50}$. For example, in rats, approximately 9,000 new cells are generated every day in the hippocampus ${ }^{51}$, and between one and two weeks after their generation, many of those cells die if they are not used in association with learning and memory ${ }^{52}$. Therefore, this period is considered critical for training in order to rescue those cells $s^{53-55}$. However, the DCX staining used in the present study labeled all cells that had been generated within the past 3 weeks ${ }^{56}$; therefore, only a portion of the cells were at the critical stage during which training is effective. We found a trend of an increase in the DCX cell count following training in the 
control group but not in the noise group. It is likely that the training effect is masked by the other cells that are not at the critical stage. Further, Ki67 staining labeled the cells that were in the phase of proliferation several hours ${ }^{57}$ before the animal was sacrificed; obviously those cells had not yet reached the critical stage for rescue. In our lab, research is being conducted to label proliferated cells using BrdU or EdU before training to verify whether NIHL reduces the rescue effect of training on neurogenesis in the hippocampus. We are also investigating whether and how NIHL impacts the expression of learning-related genes in the hippocampus.

In conclusion, the present report suggested a role of NIHL independent of its oxidative effect on cognitive function and hippocampal neurogenesis. Therefore, NIHL, if appropriately established, can be used as a model of sensorineural hearing loss to study the mechanism through which hearing loss promotes the development of dementia.

\section{Materials and Methods}

A total of $96 \mathrm{CBA} / \mathrm{CAJ}$ mice were obtained from the Experimental Animal Center of Shanghai Super-B\&K Laboratory Animal Corp. Ltd., Shanghai, China. The subjects in the noise group were exposed to an intense broadband noise at $123 \mathrm{~dB}$ SPL for 2 hours at the age of 6 and 8 weeks, while the control subjects underwent sham exposure to the environmental change.

Blood samples were taken from 8 subjects in each group at 0, 1, 7 and 28 day(s) after noise exposure to examine serum CORT level. They were returned to the colony for further evaluation. Twenty-four subjects in the noise group were sacrificed ( 8 at each of the three time points of 1 day, 1 month and 3 months after noise exposure) for the examination of oxidative stress in the hippocampus, and 8 subjects in the control group were sacrificed at $1 \mathrm{~m}$ after the sham exposure for the same evaluation.

Three months after the noise/sham exposure, all animals remaining (32 in each group) were examined for hearing threshold by the frequency specific auditory brainstem response (ABR). In each group, the 32 subjects were further divided into trained $(n=22)$ and non-trained $(n=10)$ subgroups. The capabilities of spatial learning and memory were measured in each trained subgroup by means of a Morris water maze. Immediately after the functional test, the hippocampus was harvested for the observation of neurogenesis, and the cochleae were harvested from 12 subjects in the noise group for the evaluation of hair cell loss. All animal procedures were performed in accordance with the guidelines in the ethic protocol approved by the University Committee for Laboratory Animals of Southeast University, China (Permit number: SCXK2011-0003).

Noise exposure. The animals were unrestrained in a cage $60 \mathrm{~cm}$ below the horns of two loudspeakers, a low frequency woofer and a high frequency tweeter. Electrical Gaussian noise was delivered to the speakers after power amplification. The acoustic spectrum of the sound was distributed mainly below $20 \mathrm{kHz}$, as previously reported $^{58}$. The noise level was adjusted to $123 \mathrm{~dB}$ SPL and monitored using a $1 / 4$-inch microphone linked to a sound level meter (microphone: 2520, sound level meter: 824, from Larson Davis, Depew, NY, USA).

ABR test. For the ABR recordings, the animal was anesthetized with pentobarbital $(60 \mathrm{mg} / \mathrm{kg}$, i.p.), and body temperature maintained at $37.5-38^{\circ} \mathrm{C}$ with a thermostatic heating pad. Three subdermal needle electrodes were used to record the ABRs. The non-inverting electrode was inserted at the vertex, and the reference and grounding electrodes were placed on the two earlobes.

Hardware and software (BioSig and SigGen) from Tucker-Davis Technology (TDT system III, Alachua, FL, USA) were used for stimulus generation and bio-signal acquisition. The stimuli were played through a broadband speaker (MF1 from TDT), which was placed $10 \mathrm{~cm}$ in front of the animal's head. The evoked responses were amplified 20 times and digitized via a pre-amplifier (RA16PA) with a filter between $100-3000 \mathrm{~Hz}$. The responses were averaged 1,000 times. The thresholds were measured across frequencies from 2 to $32 \mathrm{kHz}$ with tone bursts presented at the rate of $21.1 / \mathrm{s}$. At each frequency, the test was performed in a descending sequence from $90 \mathrm{~dB}$ SPL in 5-dB steps until the ABR response disappeared.

Behavioral test. The MWM setting was identical to that described previously ${ }^{59,60}$. The pool was filled to a depth of $14 \mathrm{~cm}(0.5 \mathrm{~cm}$ over the platform $)$ with tap water at $22-24^{\circ} \mathrm{C}$. The MWM test consisted of two phases. The first one was a 5-day spatial acquisition phase in which four training trials interrupted by an interval of $10 \mathrm{~min}$ were performed each day. An acclimatization session was given on the first day, as described previously ${ }^{61}$. The animal was allowed to swim and search for the hidden platform for $60 \mathrm{~s}$ during each trial. The escape latency was defined as the time needed for the animal to locate the platform before it could stay steadily on it for more than $5 \mathrm{sec}$. The animal was guided to the platform if it could not find the platform within $60 \mathrm{~s}$. The second phase (the probe trial) was performed the next to last day of the first phase with the platform removed. Each mouse was allowed to swim for $60 \mathrm{~s}$ in the pool. The number of times the mouse went across the location of the platform was recorded as the index of memory of the platform.

Oxidative stress. Blood samples were taken from the tail vein repeatedly from 8 subjects in each group at $0,1,7$ and 28 day(s) after noise exposure to examine serum CORT level. Each sample was placed in a sodium heparin-coated tube, and $50 \mu \mathrm{l}$ of supernatant plasma was obtained from each sample after centrifugation for testing. The plasma CORT concentration was measured using a Mouse Corticosterone (CORT) ELISA Kit (CSB-E07969 m, CUSABIO, Wuhan, China), strictly following the kit instructions.

Hippocampal tissue was quickly harvested on ice from mice after decapitation and was immersed in PBS of 9 times the tissue volume for ultrasonication. Supernatant was obtained after ultracentrifugation for the tests of three common indicators of oxidative stress. Commercial test kits from Cell Biolabs (San Diego, USA) were used for measuring super oxide dismutase (SOD) activity (Superoxide Dismutase Activity Assay kit STA-340), malondialdehyde (MDA) adducts (ELISA Kit, STA-332) and reactive oxygen/nitrogen species (ROS/RNS) level (In Vitro ROS/RNS Assay Kit, STA-347). Total protein concentration was measured before the MDA test using the 
Pierce $^{\circledR}$ BCA Protein Assay Kit (23225, Thermo, USA). The ROS/RNS level was tested using a ROS/RNS-sensitive fluorescent dye, DCF $\left(2^{\prime}, 7^{\prime}\right.$-dichlorofluorescin), which was generated from the reaction of ROS/RNS with a specific probe: DCFH-DA (dichlorofluorescein diacetate). The compound is hydrolyzed to DCF, which interacts with ROS/RNS to generate fluorescence. The quantity of ROS/RNS was calculated based on the standard obtained using the known ROS/RNS contents.

Neurogenesis. The animal was deeply anesthetized with pentobarbital ( $100 \mathrm{mg} / \mathrm{kg}$, i.p.) and fixed with open-chest cardiovascular perfusion of $4 \%$ paraformaldehyde in PBS buffer followed by post-fixation in the same fixative at $4{ }^{\circ} \mathrm{C}$ for $24 \mathrm{~h}$. The brain tissue block was then immersed in $30 \%$ sucrose, dehydrated at $4{ }^{\circ} \mathrm{C}$ until it sunk to the bottom, embedded in OCT compound and then frozen in a $-80^{\circ} \mathrm{C}$ freezer. The frozen block was sliced into $25 \mu \mathrm{m}$ thick sections using a microtome (Leica Cryostat Microtome 1900, Heidelberger, Germany). One section from every 3 was chosen for further processing.

Doublecortin (DCX) staining was performed using floating sections. The selected sections were permeabilized with $0.1 \%$ Triton X-100 in PBS for $30 \mathrm{~min}$, incubated for $30 \mathrm{~min}$ in $10 \%$ donkey serum in PBS and then incubated in the primary antibody (1:300, goat polyclonal anti-DCX, sc-8066, Santa Cruz, Santa Cruz, CA, USA) overnight at $4{ }^{\circ} \mathrm{C}$. This was followed by treatment with secondary antibodies (1:500, Donkey Anti Goat IgG-H\&L $\mathrm{Cy} 3^{\circledR}$, ab6949, Abcam, Cambridge, UK) for $1 \mathrm{~h}$ at room temperature. Ki67 staining was performed on affixed tissue sections. The selected slices were placed onto lysine-treated glass slides, dried at room temperature, dipped in antigen retrieval solution (mixture of citric acid buffer), and then heated in a microwave oven. The slices were then permeabilized with $0.1 \%$ Triton X-100 in PBS for $30 \mathrm{~min}$, incubated for $30 \mathrm{~min}$ in $10 \%$ goat serum and then incubated in the primary antibody (1:300, Rabbit polyclonal to Ki67, ab66155, Abcam, Cambridge, UK) overnight at $4^{\circ} \mathrm{C}$. This was followed by incubation with secondary antibodies (1:1000, Cy2-AffiniPure Goat Anti-Rabbit IgG-H\&L, 111-225-045, Jackson ImmunoResearch, West Grove, PA, USA) for 1 h at room temperature. The numbers of DCX- and Ki67-positive cells were counted under a fluorescence microscope (OLYMPUS BX53, Tokyo, Japan) and multiplied by 3 to yield the total number of DCX/Ki67-positive cells in the whole DG region in each animal brain.

SDH staining for hair cell count. The mouse was anaesthetized (sodium pentobarbital, $100 \mathrm{mg} / \mathrm{kg}, \mathrm{i} . \mathrm{m}$.) and decapitated. The otocyst was quickly removed, and the round and oval windows were picked under an anatomical microscope. The cochlea was perfused with the succinate dehydrogenase (SDH) staining solution, which contained $0.2 \mathrm{M}$ succinate dibasic hexahydrate (substrate, Sigma, St. Louis, USA) and 0.1\% Tetranitro Blue Tetrazolium Chloride (Nitro-BT, Sigma, St. Louis, USA) in $0.2 \mathrm{M}$ PBS. Then, the cochlea was immersed in the solution for $45 \mathrm{~min}$ at $37^{\circ} \mathrm{C}$ before being transferred to $4 \%$ paraformaldehyde for $24 \mathrm{~h}$ at $4{ }^{\circ} \mathrm{C}$ and decalcified with $1 \mathrm{~N} \mathrm{HCl}$. After that, the organ of Corti was dissected and mounted with $80 \%$ glycerin in PBS on a glass slide. It was observed under a light microscope, and the missing hair cells were counted to establish a cochleogram ${ }^{4}$.

Statistics. All statistical analyses were performed using SigmaPlot 12.2 software. The ANOVA tests were performed before the post-hoc tests. All data are presented as the means \pm SEM, unless otherwise specified. Significance is accepted at the level of $\mathrm{p}<0.05$.

\section{References}

1. Tucci, D., Merson, M. H. \& Wilson, B. S. A summary of the literature on global hearing impairment: current status and priorities for action. Otol Neurotol 31, 31-41 (2010).

2. Agrawal, Y., Platz, E. A. \& Niparko, J. K. Prevalence of hearing loss and differences by demographic characteristics among US adults: data from the National Health and Nutrition Examination Survey, 1999-2004. Arch Intern Med 168, 1522-1530, doi: 10.1001/ archinte.168.14.1522 (2008).

3. Cruickshanks, K. J. et al. Prevalence of hearing loss in older adults in Beaver Dam, Wisconsin. The Epidemiology of Hearing Loss Study. Am J Epidemiol 148, 879-886 (1998).

4. Nelson, D. I., Nelson, R. Y., Concha-Barrientos, M. \& Fingerhut, M. The global burden of occupational noise-induced hearing loss. American journal of industrial medicine 48, 446-458, doi: 10.1002/ajim.20223 (2005).

5. Basner, M. et al. Auditory and non-auditory effects of noise on health. Lancet 383, 1325-1332, doi: 10.1016/S0140-6736(13)61613-X (2014).

6. Cheng, L., Wang, S. H., Chen, Q. C. \& Liao, X. M. Moderate noise induced cognition impairment of mice and its underlying mechanisms. Physiol Behav 104, 981-988, doi: 10.1016/j.physbeh.2011.06.018 (2011).

7. Cui, B., Wu, M. \& She, X. Effects of chronic noise exposure on spatial learning and memory of rats in relation to neurotransmitters and NMDAR2B alteration in the hippocampus. Journal of occupational health 51, 152-158 (2009).

8. Jauregui-Huerta, F. et al. Chronic exposure of juvenile rats to environmental noise impairs hippocampal cell proliferation in adulthood. Noise Health 13, 286-291, doi: 10.4103/1463-1741.82961 (2011).

9. Wright, B., Peters, E., Ettinger, U., Kuipers, E. \& Kumari, V. Understanding noise stress-induced cognitive impairment in healthy adults and its implications for schizophrenia. Noise Health 16, 166-176, doi: 10.4103/1463-1741.134917 (2014).

10. Helfer, T. M. et al. Noise-induced hearing injury and comorbidities among postdeployment U.S. Army soldiers: April 2003-June 2009. Am J Audiol 20, 33-41, doi: 10.1044/1059-0889(2011/10-0033) (2011).

11. Cave, K. M., Cornish, E. M. \& Chandler, D. W. Blast injury of the ear: clinical update from the global war on terror. Military medicine 172, 726-730 (2007).

12. Belanger, H. G., Kretzmer, T., Yoash-Gantz, R., Pickett, T. \& Tupler, L. A. Cognitive sequelae of blast-related versus other mechanisms of brain trauma. J Int Neuropsychol Soc 15, 1-8, doi: 10.1017/S1355617708090036 (2009).

13. Chengzhi, C. et al. Recovery of chronic noise exposure induced spatial learning and memory deficits in young male Sprague-Dawley rats. Journal of occupational health 53, 157-163 (2011).

14. Hirano, Y. et al. Effect of unpleasant loud noise on hippocampal activities during picture encoding: an fMRI study. Brain Cogn 61, 280-285, doi: 10.1016/j.bandc.2006.02.003 (2006).

15. Kraus, K. S. \& Canlon, B. Neuronal connectivity and interactions between the auditory and limbic systems. Effects of noise and tinnitus. Hear Res 288, 34-46, doi: 10.1016/j.heares.2012.02.009 (2012).

16. Yu, Y. F., Zhai, F., Dai, C. F. \& Hu, J. J. The relationship between age-related hearing loss and synaptic changes in the hippocampus of C57BL/6J mice. Exp Gerontol 46, 716-722, doi: 10.1016/j.exger.2011.04.007 (2011). 
17. Kraus, K. S. et al. Noise trauma impairs neurogenesis in the rat hippocampus. Neuroscience 167, 1216-1226, doi: 10.1016/j. neuroscience.2010.02.071 S0306-4522(10)00305-2 (2010).

18. Cui, B., Wu, M., She, X. \& Liu, H. Impulse noise exposure in rats causes cognitive deficits and changes in hippocampal neurotransmitter signaling and tau phosphorylation. Brain Res 1427, 35-43, doi: S0006-8993(11)01540-X10.1016/j. brainres.2011.08.035 (2011).

19. Xu, J., Yu, L., Cai, R., Zhang, J. \& Sun, X. Early continuous white noise exposure alters auditory spatial sensitivity and expression of GAD65 and GABAA receptor subunits in rat auditory cortex. Cereb Cortex 20, 804-812, doi: 10.1093/cercor/bhp143 (2010).

20. Rabat, A., Bouyer, J. J., George, O., Le Moal, M. \& Mayo, W. Chronic exposure of rats to noise: relationship between long-term memory deficits and slow wave sleep disturbances. Behav Brain Res 171, 303-312, doi: S0166-4328(06)00210-5 10.1016/j. bbr.2006.04.007 (2006).

21. Stansfeld, S. A. et al. Aircraft and road traffic noise and children's cognition and health: a cross-national study. Lancet 365, 1942-1949, doi: S0140-6736(05)66660-3 10.1016/S0140-6736(05)66660-3 (2005).

22. Haines, M. M., Stansfeld, S. A., Job, R. F., Berglund, B. \& Head, J. Chronic aircraft noise exposure, stress responses, mental health and cognitive performance in school children. Psychol Med 31, 265-277 (2001).

23. Arnsten, A. F. \& Goldman-Rakic, P. S. Noise stress impairs prefrontal cortical cognitive function in monkeys: evidence for a hyperdopaminergic mechanism. Arch Gen Psychiatry 55, 362-368 (1998).

24. Manikandan, S. et al. Effects of chronic noise stress on spatial memory of rats in relation to neuronal dendritic alteration and free radical-imbalance in hippocampus and medial prefrontal cortex. Neurosci Lett 399, 17-22, doi: 10.1016/j.neulet.2006.01.037 (2006).

25. Schoenfeld, T. J. \& Gould, E. Stress, stress hormones, and adult neurogenesis. Exp Neurol 233, 12-21, doi: S0014-4886(11)00022-7 10.1016/j.expneurol.2011.01.008 (2012).

26. Li, W. Z. et al. Glucocorticoids increase impairments in learning and memory due to elevated amyloid precursor protein expression and neuronal apoptosis in 12-month old mice. Eur J Pharmacol 628, 108-115, doi: 10.1016/j.ejphar.2009.11.045 (2010).

27. Schoenfeld, T. J. \& Gould, E. Differential effects of stress and glucocorticoids on adult neurogenesis. Curr Top Behav Neurosci 15, 139-164, doi: 10.1007/7854_2012_233 (2013).

28. Wang, Q. et al. Glucocorticoid receptor protein expression in human hippocampus; stability with age. Neurobiol Aging 34, 1662-1673, doi: 10.1016/j.neurobiolaging.2012.11.019 (2013).

29. Lucassen, P. J. et al. Neuropathology of stress. Acta Neuropathol 127, 109-135, doi: 10.1007/s00401-013-1223-5 (2014).

30. Lucassen, P. J. et al. Regulation of Adult Neurogenesis and Plasticity by (Early) Stress, Glucocorticoids, and Inflammation. Cold Spring Harb Perspect Biol 7, doi: 10.1101/cshperspect.a021303 (2015)

31. Hunter, R. G., Gagnidze, K., McEwen, B. S. \& Pfaff, D. W. Stress and the dynamic genome: Steroids, epigenetics, and the transposome. Proc Natl Acad Sci USA 112, 6828-6833, doi: 10.1073/pnas.1411260111 (2015).

32. Lucassen, P. J. et al. Stress, depression and hippocampal apoptosis. CNS Neurol Disord Drug Targets 5, 531-546 (2006).

33. Snyder, J. S., Soumier, A., Brewer, M., Pickel, J. \& Cameron, H. A. Adult hippocampal neurogenesis buffers stress responses and depressive behaviour. Nature 476, 458-461, doi: 10.1038/nature10287 (2011).

34. Opendak, M. \& Gould, E. New neurons maintain efficient stress recovery. Cell Stem Cell 9, 287-288, doi: 10.1016/j.stem.2011.09.003 (2011).

35. Samson, J. et al. Noise-induced time-dependent changes in oxidative stress in the mouse cochlea and attenuation by D-methionine. Neuroscience 152, 146-150, doi: 10.1016/j.neuroscience.2007.11.015 (2008).

36. Sarah Hayes, S. M., Brian Allman \& Richard Salvi. Stress Hormone Levels and Hippocampal Neurogenesis Following Acoustic Trauma in The 34th Annual Midwinter meeting of Association For Research in Otolaryngology. Baltimore, USA. Association For Research in Otolaryngology (2011).

37. Zheng, Y., Hamilton, E., Begum, S., Smith, P. F. \& Darlington, C. L. The effects of acoustic trauma that can cause tinnitus on spatial performance in rats. Neuroscience 186, 48-56, doi: 10.1016/j.neuroscience.2011.04.052 (2011).

38. Lin, F. R. et al. Hearing loss and incident dementia. Arch Neurol 68, 214-220, doi: 68/2/214 10.1001/archneurol.2010.362 (2011).

39. Lin, F. R. et al. Hearing loss and cognition in the Baltimore Longitudinal Study of Aging. Neuropsychology 25, 763-770, doi: 201113415-001 10.1037/a0024238 (2011).

40. Lin, F. R. Hearing loss and cognition among older adults in the United States. J Gerontol A Biol Sci Med Sci 66, 1131-1136, doi: 10.1093/gerona/glr115 glr115 (2011).

41. Lin, F. R. et al. Hearing Loss and Cognitive Decline in Older Adults. JAMA Intern Med. 1-7, doi: 1558452 10.1001/ jamainternmed.2013.1868 (2013)

42. Moxon, K. A. et al. Multiple single units and population responses during inhibitory gating of hippocampal auditory response in freely-moving rats. Brain Res 825, 75-85 (1999).

43. O'Mara, S. The subiculum: what it does, what it might do, and what neuroanatomy has yet to tell us. J Anat 207, 271-282, doi: 10.1111/j.1469-7580.2005.00446.x (2005).

44. Shi, L. et al. Ribbon synapse plasticity in the cochleae of Guinea pigs after noise-induced silent damage. PLoS One 8, e81566, doi: 10.1371/journal.pone.0081566 PONE-D-13-30022 (2013).

45. Liu, L. et al. Silent damage of noise on cochlear afferent innervation in guinea pigs and the impact on temporal processing. PLoS One 7, e49550, doi: 10.1371/journal.pone.0049550 PONE-D-12-26008 (2012).

46. Kujawa, S. G. \& Liberman, M. C. Adding insult to injury: cochlear nerve degeneration after "temporary" noise-induced hearing loss. J Neurosci 29, 14077-14085, doi: 29/45/14077 10.1523/JNEUROSCI.2845-09.2009 (2009).

47. Kim, H. et al. Influence of prenatal noise and music on the spatial memory and neurogenesis in the hippocampus of developing rats. Brain Dev 28, 109-114, doi: 10.1016/j.braindev.2005.05.008 (2006).

48. Curlik, D. M. 2nd, Maeng, L. Y., Agarwal, P. R. \& Shors, T. J. Physical skill training increases the number of surviving new cells in the adult hippocampus. PLoS One 8, e55850, doi: 10.1371/journal.pone.0055850 PONE-D-12-21761 (2013).

49. Xu, Z. et al. Working memory task decreases the survival of newly born neurons in hippocampus. Neurobiol Learn Mem 95, 239-247, doi: S1074-7427(10)00199-1 10.1016/j.nlm.2010.11.013 (2011).

50. Anderson, M. L., Sisti, H. M., Curlik, D. M. 2nd \& Shors, T. J. Associative learning increases adult neurogenesis during a critical period. Eur J Neurosci 33, 175-181, doi: 10.1111/j.1460-9568.2010.07486.x (2011).

51. Cameron, H. A. \& McKay, R. D. Adult neurogenesis produces a large pool of new granule cells in the dentate gyrus. J Comp Neurol 435, 406-417 (2001).

52. Attardo, A. et al. Tis21 expression marks not only populations of neurogenic precursor cells but also new postmitotic neurons in adult hippocampal neurogenesis. Cerebral cortex 20, 304-314, doi: 10.1093/cercor/bhp100 (2010).

53. Veyrac, A. et al. Zif268/egr1 gene controls the selection, maturation and functional integration of adult hippocampal newborn neurons by learning. Proc Natl Acad Sci USA 110, 7062-7067, doi: 1220558110 10.1073/pnas.1220558110 (2013).

54. Epp, J. R., Chow, C. \& Galea, L. A. Hippocampus-dependent learning influences hippocampal neurogenesis. Front Neurosci 7, 57, doi: 10.3389/fnins.2013.00057 (2013).

55. Gould, E., Beylin, A., Tanapat, P., Reeves, A. \& Shors, T. J. Learning enhances adult neurogenesis in the hippocampal formation. Nat Neurosci 2, 260-265, doi: 10.1038/6365 (1999).

56. Kohler, S. J., Williams, N. I., Stanton, G. B., Cameron, J. L. \& Greenough, W. T. Maturation time of new granule cells in the dentate gyrus of adult macaque monkeys exceeds six months. Proc Natl Acad Sci USA 108, 10326-10331, doi: 10.1073/pnas. 1017099108 (2011). 
57. Bruno, S. \& Darzynkiewicz, Z. Cell cycle dependent expression and stability of the nuclear protein detected by Ki- 67 antibody in HL-60 cells. Cell proliferation 25, 31-40 (1992).

58. Wang, J. et al. Overexpression of X-linked inhibitor of apoptosis protein protects against noise-induced hearing loss in mice. Gene Ther 18, 560-568, doi: gt2010172 10.1038/gt.2010.172 (2011).

59. Mendez-Couz, M., Conejo, N. M., Vallejo, G. \& Arias, J. L. Spatial memory extinction: A c-Fos protein mapping study. Behav Brain Res 260, 101-110, doi: 10.1016/j.bbr.2013.11.032 S0166-4328(13)00719-5 (2014).

60. Bonaccorsi, J. et al. System consolidation of spatial memories in mice: effects of enriched environment. Neural Plast 2013, 956312, doi: $10.1155 / 2013 / 956312(2013)$.

61. Vorhees, C. V. \& Williams, M. T. Morris water maze: procedures for assessing spatial and related forms of learning and memory. Nat Protoc 1, 848-858, doi: nprot.2006.116 10.1038/nprot.2006.116 (2006).

\section{Acknowledgements}

This study was supported by a grant from Nature Science Foundation of China (Grant\#: 81272086) and by a grant from Natural Science and Engineering Research Council of Canada (Grant\#: RGPIN-2014-05437). With thanks to Dr. Richard Brown for his instruction on the behavior test.

\section{Author Contributions}

L.L., P.S. and T.H. conducted experiment and data analysis. L.S. conducted data analysis and paper writing. S.T., X.L. Q.X., X.G., Z.Y. and Y.C. conducted sample processing and data collection. J.W. conducted research design, data analysis and paper writing. All authors reviewed the manuscript.

\section{Additional Information}

Competing financial interests: The authors declare no competing financial interests.

How to cite this article: Liu, L. et al. Noise induced hearing loss impairs spatial learning/memory and hippocampal neurogenesis in mice. Sci. Rep. 6, 20374; doi: 10.1038/srep20374 (2016).

(c) (i) This work is licensed under a Creative Commons Attribution 4.0 International License. The images or other third party material in this article are included in the article's Creative Commons license, unless indicated otherwise in the credit line; if the material is not included under the Creative Commons license, users will need to obtain permission from the license holder to reproduce the material. To view a copy of this license, visit http://creativecommons.org/licenses/by/4.0/ 